\title{
Development of the production cost calculating method in complex industries of the agro- industrial complex
}

\author{
Nikolay Chernegov ${ }^{1, *}$, Alexander Tatochenko ${ }^{1}$, Irina Tatochenko $^{1}$, Taisiya Ryabova $^{1}$, and \\ Larisa Poletaeva ${ }^{1}$ \\ ${ }^{1}$ K.G. Razumovsky Moscow State University of Technologies and Management (the First Cossack \\ University), 73, Zemlyanoy val, Moscow, 109004, Russia
}

\begin{abstract}
This study solves the most important economic problem of developing a method for calculating the cost of each type of product in complex and multi-product industries. The performed analysis of the current legislation and the methods used for calculating the cost has led to the conclusion that today the solution to this problem has no scientific justification. The authors carried out a systematic development of procedures that allow building a scientific idea using objective information. It is implemented in the study by means of appropriate techniques and the construction of an economic and mathematical model that allows the development of an instrumental apparatus for the implementation of the method. With its application, the problem of calculating the production cost of seven types of final products for a conditional technological scheme of integrated production was solved. The results are also compared with the cost calculation obtained by other methods. In addition, a graphical and matrix analysis of the calculation results was carried out. The obtained results of calculating the cost of the final product make it possible to evaluate this method as workable, and the algorithm for its construction - as using the entire array of initial objective information.
\end{abstract}

\section{Introduction}

The Russian economy development in recent years is directly related to the expansion of the material base and the intensification of domestic production. This orientation inevitably requires a significant amount of processing of raw materials, and this applies to many sectors and sectors of the economy. Among them are the basic industries: mechanical engineering, the agro-industrial complex, as well as the food and processing industry, processing of resources and, as a result, waste disposal.

In general, this vector of economic development is undoubtedly promising, but it can cause negative consequences. Thus, the large-scale use of resources has already led the economy of our country to extensive development. The reason for this is well known. An increase in the volume of production does not always lead to an increase in labor

${ }^{*}$ Corresponding author: chernick@mail.ru 
productivity and an increase in the efficiency of enterprises. It often entails a significant amount of waste and inefficient use of secondary resources. However, according to many experts, the integrated use of raw materials allows the total volume of waste in various sectors of the economy from $25 \%$ to $50 \%$. [1] Therefore, the task of creating resourcesaving and waste-free technologies is currently recognized as one of the most important. It is solved through many tools and methods - organizational, technological, legislative and economic.

\section{Problem Statement}

One of the most important economic tasks of complex and multi-product industries, which today do not have a single and reliable solution, is to determine the cost of each type of product. At the same time, the calculation of the costs for the release of each type of product allows you to set prices, determine the profit from its sale, and justify the optimal list of the company's products. In addition, if the costs of producing a certain product turn out to be high and do not allow making a profit, the company can make technological or organizational changes in its production to reduce costs and in some cases refuse to release it.

In general, the task of calculating costs in complex industries is as follows. Part of the costs of production are direct, that is, attributable to the production of each type of final product, which is due to the technology of its production. Along with this, there are general costs that cannot be directly attributed to the cost of each type of product. This is shown on a conditional technological scheme of integrated production of seven types of products (Fig. 1), where costs are given in conventional units.

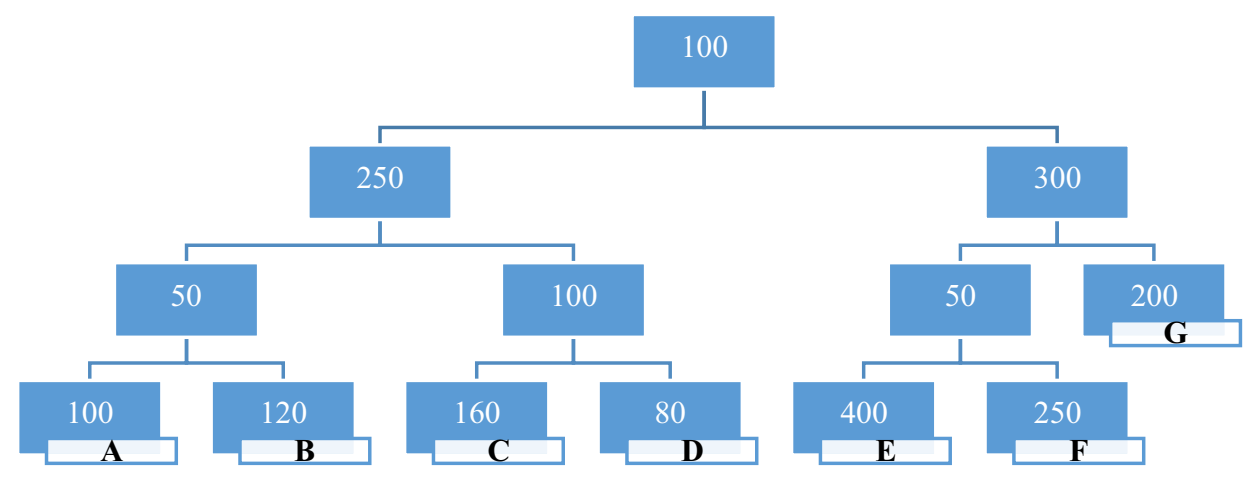

Fig. 1. Conditional technological scheme of production of seven types of products.

Thus, if the costs of producing each type of final product (hereinafter referred to as each component) can be directly attributed to its cost, then the distribution of total costs (for all or several components) does not have a clear economic justification.

The Tax Code of the Russian Federation regulates the process of grouping expenses as follows: "If some costs with equal grounds can be simultaneously attributed to several groups of expenses, the taxpayer has the right to independently determine which group he will attribute such costs to." (Article 252 of the Tax Code of the Russian Federation (part two) of 05.08.2000 N 117-FZ). Thus, the method of calculating the cost price is not legally regulated, and any enterprise must independently decide how to perform the calculation. 


\section{Materials and Methods}

In scientific research and in practice, the following main approaches to calculating costs in complex and multi-product industries have developed.

Cost elimination method, in which all products are divided into main and associated products. The cost of the main product is determined by the difference between the total cost and the cost of by-product. The latter are calculated by direct costs associated with the release of those products that are considered incidental. The advantage of the method is its ease of use. The disadvantage is that the part of the total costs actually participating in the formation of costs for by-products is not taken into account when calculating its cost. In addition, the division of products into main and associated products is in some cases subjective. [2]

A cost allocation method in which the direct costs of producing each component are charged to its cost, and the total costs are allocated to the components according to various criteria. They can be technical indicators (volume, commodity weight, etc.) or economic (price, labor intensity, etc.). The disadvantage is the subjective approach to the selection of the distribution criterion, which does not have a clear economic justification. [3]

Combined methods that include elements of shutdown and distribution methods. [4]

Direct costing method is based on dividing costs into direct and fixed costs. The main problem of the method is the difficulty in determining and differentiating variable and fixed costs, since very often in practice they are difficult to classify and refer to a specific group. [5]

Other methods of calculating the cost in complex industries are also used - standard cost [6], absorption costing [7], target costing [8], activity-based costing [9] and other methods [10]. They contain the merits and demerits of shutdown and distribution methods and have no business rationale. As a result, it is impossible to accurately calculate the cost of each component, set a price for it, and determine the profit from sales. In addition, in a number of cases it is also necessary to establish the feasibility of producing such a set of types of products that allows the enterprise to receive the greatest profit.

This study proposes a cost calculation method that is free from the drawbacks of existing methods [11]. As basis there were taken two approaches, not related to the introduction of subjective information and the conventionality of dividing products into the main and associated. The technological scheme of a production complex was considered not as an object for finding a criterion for the distribution of total costs, but, first, as a technological and economic system, which is an object for calculating the totality of costs.

The first approach was developed in the 70s of the twentieth century at the Research Institute for Pricing of the State Committee for Prices of the Council of Ministers of the USSR (hereinafter - RIpr). The second - State Research Center Federal State Unitary Enterprise "Central Research Institute of Ferrous Metallurgy named after I.P. Bardeen" (hereinafter - RICfm). Additional information is created by the introduction of the concepts "incomplete complex", "full complex". A full complex of production means its technological scheme with an indication of the costs for all redistributions, calculated according to the established nomenclature of production costs. An incomplete complex is only modeled [12] and differs from the complete absence of technological processes associated with the release of one or several types of products.

In the RIpr method, an incomplete complex is understood as the totality of the technological scheme and costs at which the production of the (n-1)-th type of final product is carried out, where $\mathrm{n}$ is the number of types of final product. By means of artificial sequential disconnection from a complete complex of each type of final product, a system of complete and incomplete complexes without one component is formed. The number of incomplete complexes corresponds to the number of types of final products. Known and 
true are only the total costs of the full complex, denoted by $a$. By sequentially disconnecting each component from the complete complex with its direct costs, a system of equations is created, where $X^{\prime}{ }_{i}$ - the values of the total individual costs for the release of the component, calculated using the system of incomplete complexes. The number of equations is equal to the number of unknowns:

$$
\left\{\begin{array}{c}
X_{1}+X_{2}+\cdots+X_{n}=a=\sum_{i=1}^{n} X_{i} \\
X_{2}^{\prime}+X^{\prime}{ }_{3}+\cdots+X_{n-1}^{\prime}+X^{\prime}{ }_{n}=a_{1} \\
X_{1}^{\prime}{ }_{1}+\ldots+X_{3}+\cdots+X^{\prime}{ }_{n-1}+X^{\prime}{ }_{n}=a_{2} \\
X^{\prime}{ }_{1}+X^{\prime}{ }_{2}+\cdots+X^{\prime}{ }_{n-1}+X^{\prime}{ }_{n}=a_{3} \\
\cdots \cdots \cdots \cdots \cdots \cdots \cdots \cdots \cdots \cdots \cdots \cdots \cdots \cdots \cdots \cdots \cdots \\
X^{\prime}{ }_{1}+X^{\prime}{ }_{2}+X^{\prime}{ }_{3}+\cdots+X^{\prime}{ }_{n-1}+X^{\prime}{ }_{n}=a_{n} \\
X^{\prime}{ }_{1}+X^{\prime}{ }_{2}+X^{\prime}{ }_{3}+\cdots X^{\prime}{ }_{n-2}+X^{\prime}{ }_{n-1}=a_{n}
\end{array}\right.
$$

Such a system has a fairly rigorous mathematical solution.

However, as a result of the calculation, the sum of the values of the total individual costs calculated using the indicators of the system of incomplete complexes is greater than the production costs of the full complex. This is due to the fact that in incomplete complexes the same attributable costs are posted to a smaller number of components. The amendment can be made in two ways.

1. The difference in the sums of costs calculated for the full complex and through the system of full and incomplete complexes is divided by the number of components and subtracted from the individual costs found for each component:

$$
\left\{\begin{array}{c}
X_{i}=\frac{1}{n}-1 \sum_{i=1}^{n} a_{i}-a_{i} \\
b=\frac{1}{n}-1 \sum_{i=1}^{n} a_{i} \\
X^{\prime}{ }_{i}=b-a_{i} \\
\Delta=b-a_{i}
\end{array}\right.
$$

2. The difference in the amount of costs is distributed among all components in proportion to the calculated values of the total individual costs obtained from the system of complete and incomplete complexes:

$$
\left\{\begin{array}{l}
X_{i}=X^{\prime}{ }_{i}+t \\
X_{i}=X^{\prime}{ }_{i}-\frac{\Delta}{n} \\
\text { when } t=\frac{\Delta}{n}
\end{array}\right.
$$

The method developed at RICfm is similar, but involves the creation of incomplete complexes from one component. The total amount of costs is distributed in proportion to the individual costs established through a system of incomplete complexes, each of which contains one component.

\section{Results}

Analyzing the approach to the number of components to be disconnected, one can see that the RIpr and RICfm methods use only a fraction of the objective economic information. Only incomplete complexes consisting of one component and incomplete complexes without one component are considered. However, in the general case, there is a set of complete complexes with $\mathrm{k}=1,2, \ldots,(\mathrm{n}-1)$. Suppose that both methods can serve as a basis and be part of a more general method that considers the entire system of incomplete complexes. If we take this idea as a basis, first, it is necessary to identify the number and 
composition of incomplete complexes, as well as the value of the costs for each component. For the given conditional technological scheme of seven components, this problem was solved by the formation of all incomplete complexes, united into groups according to the number of disconnected components.

The second important stage in the development of the method consists in calculating the costs in each incomplete complex and their groups, combined by the number of components. This is necessary to use the obtained data on the values of the costs for each component, depending on the number of components to be disabled. For the given technological scheme, such a calculation was also performed.

At the third step of the idea, the following qualitative-quantitative relationship is used, which was an element of the hypothesis when developing the method. It consists in the fact that with an increase in the number of disconnected components, the share of costs attributable to each final component in the technological scheme increases. Thus, monotonous dependences of costs on the number of disconnected components are formed, presented in table 1 and fig. 2, 3, 4 .

Table 1. Dependence of component costs on the number of components for a system of incomplete complexes.

\begin{tabular}{|c|c|c|c|c|c|c|}
\hline \multirow{2}{*}{ Component } & \multicolumn{6}{|c|}{ Component costs depending on their number in incomplete complexes. conv. } \\
\cline { 2 - 7 } & 6 & 5 & 4 & 3 & 2 & 1 \\
\hline & 241.7 & 249.5 & 297 & 306 & 454 & 550 \\
\hline A & 261.7 & 269.5 & 303 & 332 & 448 & 570 \\
\hline B & 301.7 & 305.3 & 363 & 391 & 628 & 660 \\
\hline C & 221.7 & 234.1 & 286 & 313 & 480 & 580 \\
\hline D & 541.7 & 553.8 & 617 & 640 & 704 & 800 \\
\hline E & 391.7 & 437.5 & 459 & 507.6 & 478 & 650 \\
\hline F & 341.7 & 343.5 & 401 & 486 & 550 & 475 \\
\hline G & 2302 & 2393 & 2726 & 2976 & 3742 & 4285 \\
\hline Total costs: & & & & & \\
\hline
\end{tabular}

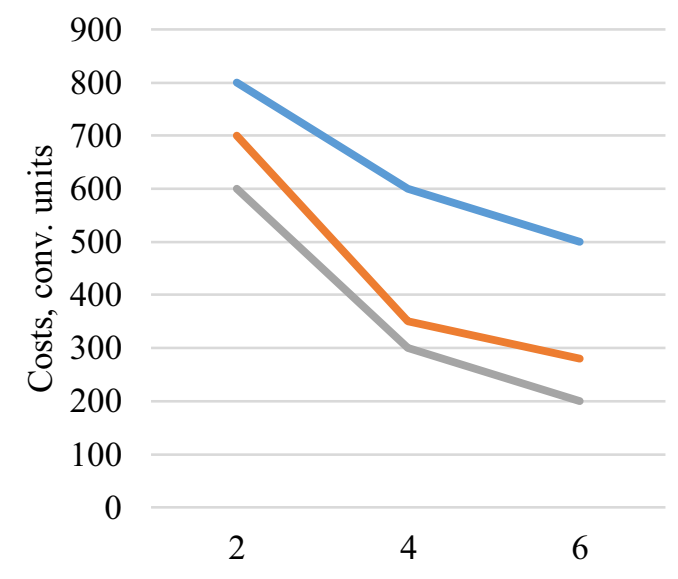

The number of components in the $-5 \stackrel{\text { complex }}{-}-1$

Fig. 2. Cost values depending on the number of disconnected components for products A, C, E. 


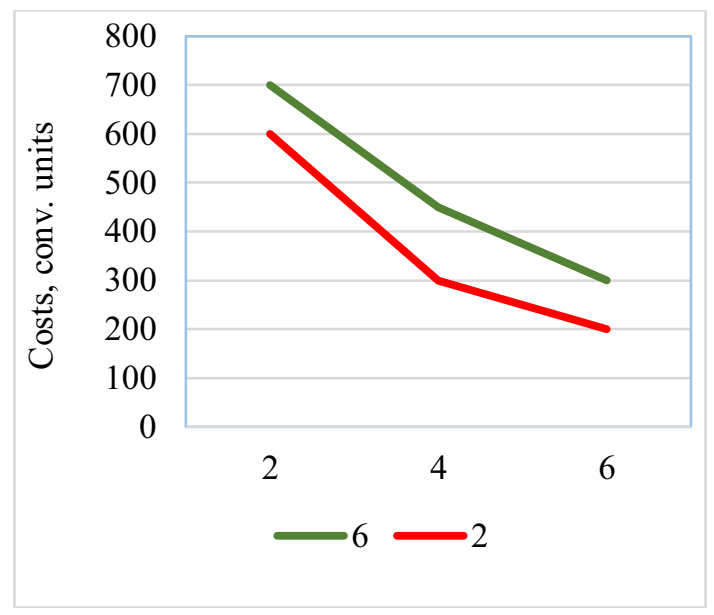

Fig. 3. Cost values depending on the number of disconnected components for products B, F.

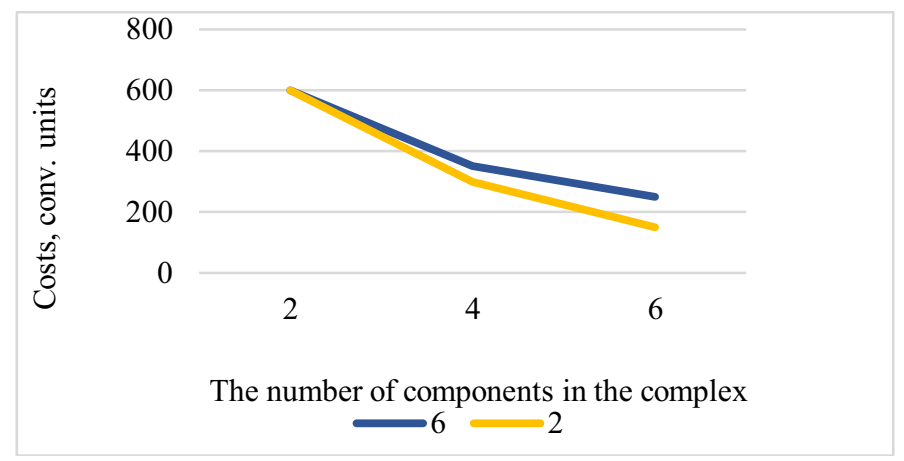

Fig. 4. Cost values depending on the number of disconnected components for products D, G.

\section{Discussion}

Thus, the indicated dependence is shown in the economic logic and the hypothesis of the method, and the results of calculations have confirmed its existence. Consequently, the required value of the costs for each component in a complete complex can be obtained by extrapolating this dependence to the number of types of final products in a complete complex. Conventionally, this situation can be called "zero outage" or the model [13] of a full complex with the establishment of costs for each component. These values will be the calculated production cost of each type of product.

When extrapolating the revealed dependence, it is necessary to take into account the equality of the sum of extrapolated values of costs (received individual costs) to the sum of costs for the full range of production. If this condition was not included in the problem, it would be possible to extrapolate the dependences of costs in incomplete complexes on the number of disconnected components using standard methods of mathematical statistics. [14] But in connection with this condition, it was necessary to develop a mathematical apparatus for the implementation of the economic idea of extrapolation of dependencies. It synthesizes Lagrange least squares and least squares methods for a set of dependencies. 


$$
\begin{gathered}
\left\{\begin{array}{c}
Y=f_{1}\left(X_{i}\right) ;(i=1,2, \ldots, n), \\
Y=f_{2}\left(X_{i}\right) ;(i=1,2, \ldots, n), \\
\cdot \\
\cdot \\
\dot{y} \\
Y=f_{1}\left(X_{i)} ;(i=1,2, \ldots, n),\right. \\
\text { at } \sum Y_{0}=f_{i}\left(X_{0}\right)=a
\end{array}\right. \\
Y_{i}=f_{i}\left(X_{i} ; a_{i 1} ; a_{i 2} ; a_{i m}\right) \\
\left.\delta=\delta_{1}+\delta_{2}+\cdots \delta_{n}=\sum_{i=1}^{n} \sum_{k=1}^{m} f_{i}\left(x ; a_{i 1}, a_{i 2}, \ldots, a_{i m}\right)\right]^{2} \\
Y_{i o=}=f_{i}\left(X_{i} ; a_{i 1} ; a_{i 2} ; a_{i m}\right)
\end{gathered}
$$

Using the developed approach, the cost of production of the products presented in the technological scheme was calculated (Fig. 1). The results of calculating the cost of seven types of final products, also performed using the RIpr and RICfm methods, are presented in table 2.

Table 2. The results of calculating costs by type of product.

\begin{tabular}{|l|c|c|c|c|c|c|c|}
\hline \multirow{2}{*}{ Method } & \multicolumn{7}{|c|}{ Component production cost } \\
\cline { 2 - 8 } & A & B & C & D & E & F & G \\
\hline RIpr & 225 & 294 & 281 & 206 & 504 & 365 & 318 \\
\hline RICfm & 275 & 285 & 330 & 290 & 400 & 325 & 240 \\
\hline The proposed & 207 & 227 & 236 & 170 & 539 & 438 & 363 \\
\hline
\end{tabular}

The cost calculation results obtained by different methods differ. In this case, the closest solutions are obtained by the RIpr method and the proposed method.

\section{Conclusion}

Thus, as a result of the research carried out, methods for calculating the cost of production in complex and multi-product industries have been analyzed, their features, advantages and disadvantages have been studied. It is concluded that there is an approach in the scientific community that makes it possible to introduce additional objective information into the solution of the problem. On its basis, a fundamentally new method for calculating costs in complex industries has been developed, and a representative mathematical apparatus has been applied. The peculiarities of the method have been studied and its efficiency has been tested using a conditional example. In the future, the authors intend to improve the economic and mathematical tools of the method proposed in the study, and to test it on the basis of integrated industries [15]. And the authors expect to present the calculation of the cost of production of the enterprises of the agro-industrial complex in Russia in subsequent publications.

\section{References}

1. A. Samukov, Ecology and Industry of Russia 23(7), 15-19 (2019) https://doi.org/10.18412/1816-0395-2019-7-15-19

2. N. Bozgulova, R. Parmanova, M. Abenova, et al., Entrepreneurship and Sustainability 7(2), 1450-1461 (2019) https://doi.org/10.9770/jesi.2019.7.2(46)

3. A. Dmitrenko, G. Lebedyk, M. Nesterenko, International Journal of Engineering \& Technology 7(3.2), 6-11 (2018) https://doi.org/10.14419/ijet.v7i3.2.14367 
4. M. Zarichkova, News of Pharmacy, Organization and Economy of Pharmacy 3(91), 45-60 (2017) https://doi.org/10.24959/nphj.17.2176

5. L. Korobeinikova, A. Zolototrubov, Modern Economics: Problems and Solutions, 2134 (2020) https://doi.org/10.17308/meps.2020.6/2381

6. I. Akulova, G. Slavcheva, International science and technology conference "FarEastCon-2019", IOP Conf. Series: Materials Science and Engineering 753(4) (2020) https:// doi:10.1088/1757-899X/753/5/052056

7. R. Ildarkhanov, Periodica Polytechnica Transportation Engineering 47(4), 277-282 (2019) https://doi.org/10.3311/PPtr.10553

8. I. Sarbu, V. Chirmici, ECONOMY AND SOCIOLOGY: Theoretical and Scientifical Journal, Socionet, Complexul Editorial "INCE" 3, 48-56 (2014)

9. A.Y. Sokolov, Y.M. Giniatullin, Asian Social Science 11, 99-103 (2015) https://doi.org/10.5539/ASS.V11N11P99

10. A. Lada, V. Sazonov, P. Skobelev, Indian Journal of Science and Technology 9(20), 16 (2016) https://dx.doi.org/10.17485/ijst/2016/v9i20/94478

11. O. Moshchenko, V. Rokotyanskaya, V. Matveeva, S. Romanova, S. Maryanova, Herald NAMSCA 3 ,1122-1127 (2018)

12. L. Makarevich, A. Ulezko, V. Reimer, Advances in Economics, Business and Management Research 147 (2020) DOI: 10.2991/aebmr.k.200729.070

13. R. Oganyan, N. Narakidze, D. Shaykhutdinov et al., IOP Conf. Series: Materials Science and Engineering 441(1), 1-7 (2018) doi:10.1088/1757-899X/441/1/012033

14. D. Zinchenko, A. Akimov, M. Babii, Bulletin of the Karaganda University, Mathematics Series 94, 149-159 (2019) DOI: 10.31489/2019M2/149-159

15. E.A. Pribytkov, A.B. Zolotukhin, O.T. Gudmestad, Oil and Gas Facilities 3(4), 47-53 (2014) DOI: $10.2118 / 166879-P A$ 